\title{
Pericarditis in Covid 19 - An Unusual Presentation
}

\author{
Abhijit Wadekar ${ }^{1}$, Sanyauta Hepat², Sourya Acharya ${ }^{3}$, Sunil Kumar ${ }^{4}$ \\ 1, 2, 3, 4 Department of General Medicine, Datta Meghe Institute of Medical Sciences, Wardha, Maharashtra, India.
}

\section{INTRODUCTION}

Corona virus disease-19 (COVID-19) infection causing the current pandemic began as a cluster of cases in Wuhan Province of China which has now progressed to the current dangerously exponential rise of billions of cases all over the world. ${ }^{1}$ COVID19 virus is a single stranded RNA virus having zoonotic origin. It's ability to cause cross infection is the reason for the current devastating pandemic. ${ }^{2}$ Adding to that, COVID-19 is also called as novel corona virus as such a strain of virus had never affected mankind before. The spectrum of infection is also quite interesting. Cases range from asymptomatic, mild infections having cold, cough and fever, to life threatening instances of acute respiratory failure requiring ventilator support and intensive care admission. ${ }^{3}$ With fewer treatment options available, vaccination in the pipeline, the major mode of management is symptomatic treatment. Higher antibiotics, immunity stimulant drugs, oxygen supplementation currently form the mainstay of treatment. The management of Covid-19 still remains us shooting in the dark, exploring for ground-breaking options which prevent mortality and morbidity.

Another lesser explored facet of the Covid-19 infection, is its ability to rapidly disseminate in the body and not be restricted just to the respiratory system. This rapid dissemination is the reason for the multi-systemic involvement in extreme cases of Covid-19.4 There have been reported cases of Covid-19 along with neurological and cardiovascular involvement. The cardiovascular involvement can also range from chest pain and palpitations to emergencies like cardiac tamponade and cardiac failure. As the aetiology and complete progression of this disease is yet to be fully understood, such cases with multi-systemic involvement pose imminent challenges in treatment and management. ${ }^{5}$

Here, we are presenting a case of 29-year-old male Covid positive patient, who presented with chest pain and tachycardia and diagnosed as pericarditis.

\section{PRESENTATION OF CASE}

A 29-year-old male patient came to the casualty with complains of fever and outside reverse transcription polymerase chain reaction (RT PCR) Covid-19 positive report, and was admitted in Covid positive ward with stable vitals of pulse $78 / \mathrm{min}$, BP 100/70 mg. Patient was started with standardized Covid-19 treatment protocol. On 3rd day of admission, patient started complaining of chest pain which was diffuse in nature, pain was worse on laying down and was relieved on sitting and leaning forward. On examination, patient had heart rate of $130 / \mathrm{min}$. Auscultation revealed a pericardial rub with a diastolic and pre systolic component just inside the apical area. Electrocardiography (ECG) was done showing ST segment elevation with PR depression in most of the leads as per Fig. 1.

\section{DISCUSSION OF MANAGEMENT}

2D Echo was done which was suggestive of mild to moderate pericardial effusion. With these findings, patient was diagnosed with pericarditis and was started with colchicine $0.5 \mathrm{mg}$ twice a day and ibuprofen $400 \mathrm{mg}$ twice a day after which patient showed dramatic improvement and by $7^{\text {Th }}$ day, patient was symptomatically relieved and was discharged later after.
Corresponding Author:

Dr. Sourya Acharya,

Department of General Medicine,

AVBRH, Sawangi, Wardha,

Maharashtra, India.

E-mail: souryaacharya74@gmail.com

DOI: $10.14260 /$ jemds/2021/519

How to Cite This Article:

Wadekar A, Hepat S, Acharya S, et al. Pericarditis in Covid-19 an unusual presentation. J Evolution Med Dent Sci 2021;10(31):2532-2534, DOI: 10.14260/jemds/2021/519

Submission 20-04-2021,

Peer Review 03-07-2021,

Acceptance 09-07-2021,

Published 02-08-2021.

Copyright (C) 2021 Abhijit Wadekar et al. This is an open access article distributed under Creative Commons Attribution License [Attribution 4.0 International (CC BY 4.0)] 


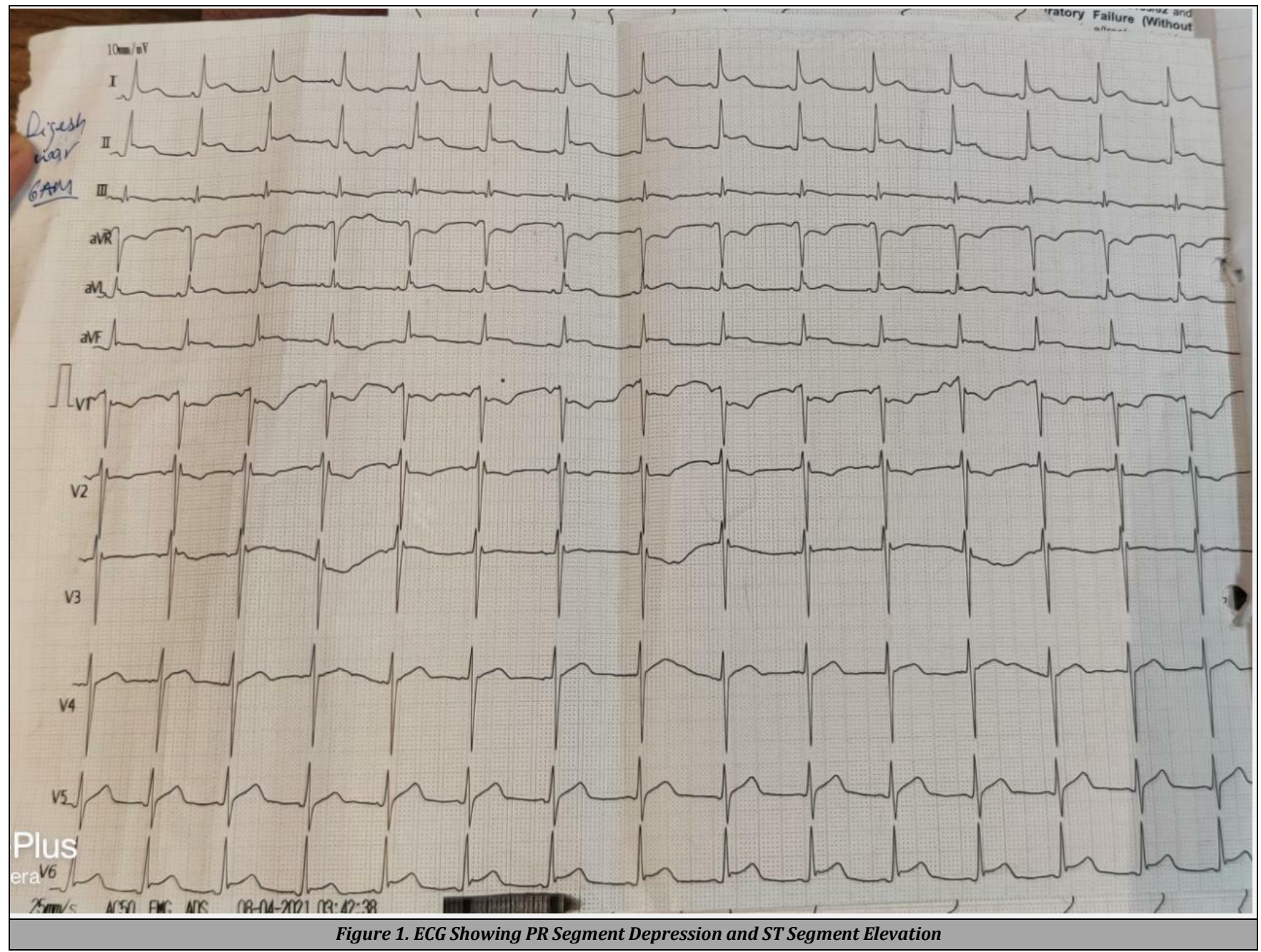

\section{DISCUSSION}

The current devastating pandemic grappling the world is caused by severe acute respiratory syndrome - coronavirus 2 (SARS - CoV-2), or Covid-19 virus. It is a single stranded RNA virus affecting various animal species having this unique ability to cross over species and affect humans. The spectrum of Covid-19 infection ranges from simple cold and cough to life threatening acute respiratory distress syndrome (ARDS), severe acute respiratory syndrome.5,6 Another worrisome aspect of Covid-19 is its ability to cause multi-systemic involvement. Covid-19 can have devastating consequences because of its ability to involve various systems of the body. ${ }^{7}$ Covid-19 infection has innumerable adverse effects on the cardiovascular system ranging from mild palpitations to severe conditions like myocarditis and cardiac failure. There are even several reported morbid cases of post infection haemorrhagic pericardial effusion which were associated with cardiac tamponade.

Pericarditis can often be described as inflammation of the pericardium which can have variable aetiology, viral infections being the most common one. Pericarditis is usually associated with pleuritic type of chest pain which gets worsened on coughing and lying down. It may or may not be associated with low grade fever, palpitations, breathlessness and generalised fatigue. Pericarditis often progresses to pericardial effusion which is accumulation of fluid between the visceral and parietal pericardium, diagnosed on transthoracic echocardiography or ECG. One of its most life-threatening complication is cardiac tamponade. ${ }^{8}$ The exact incidence of cases showing direct association between COVID-19 infection and pericarditis is still under review. The involvement of pericardium along with tamponade may or may not be associated with pulmonary involvement such as pneumonia presenting as patchy consolidation or fibrous streaks on radiological studies. ${ }^{9,10}$ There are a few cases documented in which patients with Covid-19 infection have had massive pericardial effusion which leads to tamponade and had to be dealt with therapeutic thoracentesis as mode of management. Serological testing of the pericardial fluid for Covid-19 can be done but a negative report does not necessarily rule out active Covid-19 infection leading to pericardial involvement. ${ }^{11}$

Myocardial inflammation is not an uncommon sight in Covid-19, based on troponin and cardiac MRI findings. Lifethreatening myopericarditis can occur occasionally. The degree of cardiac involvement may not be related to the severity of lung involvement. Pericardial fluid is exudative and serology can show absence of virus, therefore representing an inflammatory and not an infectious process. ${ }^{10,11}$ Furthermore, the degree of pericardial inflammatory response does not necessarily correlate with the degree of myocardial involvement. Patients with cardiac disease and/or a structurally abnormal heart may be at an increased risk of developing Covid-19 myopericarditis and cardiac tamponade. 
The pathogenesis of Covid-19 myopericarditis still remains a mystery. Two predominant mechanism theories are widely accepted. First one being, the affinity of the virus i.e., binding of SARS-CoV-2 S protein to human angiotensinconverting enzyme present in the heart, which allows room for infection. ${ }^{11}$ Secondly, myopericarditis could be a direct result of viral replication and dissemination in the blood, a week to month after the symptoms begin. This could lead to a cytokine storm and cellular inflammatory infiltration.

There is a lack of structured guidelines and evidence-based medicine outcomes regarding management of pericarditis in Covid-19. Treatment modalities are dependent on the extent of the infection and degree of involvement of the cardiovascular system. Treatment options include use of colchicine, steroids, newer drugs like tocilizumab or even therapeutic pericardiocentesis.11,12 Over the counter nonsteroidal anti-inflammatory drugs (NSAIDs) are avoided as they are sources of potential worsening of the respiratory symptoms. Corticosteroids are preferred due to their wellestablished role in tackling cytokines storm. Colchicine is also considered as a forefront in the treatment options due to its action on NLRP3 inflammasomes and cytokine's release.

\section{CONCLUSIONS}

Cardiac involvement during active Covid-19 infection or in the post Covid period should be a better explored avenue. Clinicians and physicians should conduct extensive research so as to avoid complications and well as mortality due to cardiac involvement. The atypical manifestations of Covid along with its management should be better studied especially in young patients.

Financial or other competing interests: None.

Disclosure forms provided by the authors are available with the full text of this article at jemds.com.

\section{REFERENCES}

[1] Imazio M, Klingel K, Kindermann I, et al. COVID-19 pandemic and troponin: indirect myocardial injury, myocardial inflammation or myocarditis? Heart 2020;106(15):1127-31.
[2] Lala A, Johnson KW, Januzzi JL, et al. Prevalence and impact of myocardial injury in patients hospitalized with COVID-19 infection. J Am Coll Cardiol 2020;76(5):533-46.

[3] Sala S, Peretto G, Gramegna M, et al. Acute myocarditis presenting as a reverse Tako-Tsubo syndrome in a patient with SARS-CoV-2 respiratory infection. Eur Heart J 2020;41(19):1861-62.

[4] Chen C, Zhou Y, Wang DW. SARS-CoV-2: a potential novel etiology of fulminant myocarditis. Herz 2020;45(3):23032.

[5] Tavazzi G, Pellegrini C, Maurelli M, et al. Myocardial localization of coronavirus in COVID-19 cardiogenic shock. Eur J Heart Fail 2020;22(5):911-5.

[6] Imazio M, Brucato A, Lazaros G, et al. Anti - inflammatory therapies for pericardial diseases in the COVID-19 pandemic: safety and potentiality. J Cardiovasc Med (Hagerstown) 2020;21(9):625-9.

[7] Adler Y, Charron P, Imazio M, et al. 2015 ESC Guidelines for the diagnosis and management of pericardial diseases: the task force for the diagnosis and management of pericardial diseases of the European society of cardiology (ESC) Endorsed by: the European Association for CardioThoracic Surgery (EACTS). Eur Heart J 2015;36(42):2921-64.

[8] Multicenter Collaboration Group of Department of Science and Technology of Guangdong Province and Health Commission of Guangdong Province for Chloroquine in the Treatment of Novel Coronavirus Pneumonia. Expert consensus on chloroquine phosphate for the treatment of novel coronavirus pneumonia. Zhonghua Jie He He Hu Xi Za Zhi 2020;43(3):185-8.

[9] Maestroni S, Di Corato PR, Cumetti D, et al. Recurrent pericarditis: autoimmune or autoinflammatory? Autoimmun Rev 2012;12(1):60-5.

[10] Cremer PC, Kumar A, Kontzias A, et al. Complicated pericarditis: understanding risk factors and pathophysiology to inform imaging and treatment. J Am Coll Cardiol 2016;68(21):2311-28.

[11] Wang D, Hu B, Hu C, et al. Clinical characteristics of 138 hospitalized patients with 2019 novel coronavirusinfected pneumonia in Wuhan, China. JAMA 2020;323(11):1061-9.

[12] Li K, Wu J, Wu F, et al. The clinical and chest CT features associated with severe and critical COVID-19 pneumonia. Invest Radiol 2020;55(6):327-31. 